INFLAMMATORY BOWEL DISEASE

\title{
Randomised controlled trial of azathioprine and 5 -aminosalicylic acid for treatment of steroid dependent ulcerative colitis
}

\author{
S Ardizzone, G Maconi, A Russo, V Imbesi, E Colombo, G Bianchi Porro
}

Gut 2006;55:47-53. doi: 10.1136/gut.2005.068809

See end of article for authors' affiliations

Correspondence to: Dr S Ardizzone, Cattedra di Gastroenterologia, Azienda Ospedaliera " $\mathrm{L}$ Sacco", Polo Universitario, Via G.B. Grassi, 74, 20157 Milano, Italy; samaril@tin.it

Revised version received 11 May 2005

Accepted for publication 31 May 2005

Published online first

21 June 2005
Background and aim: There are limited evidence based data concerning the use of azathioprine in ulcerative colitis. We aimed to compare the efficacy of azathioprine and oral 5-aminosalicylic acid in inducing remission of steroid dependent ulcerative colitis.

Methods: Seventy two patients with steroid dependent ulcerative colitis were admitted to this investigatorblind study. Steroid dependence was defined as a requirement for steroid therapy $\geqslant 10 \mathrm{mg} /$ day during the preceding six months, with at least two attempts to discontinue the medication. The disease had to be clinically and endoscopically active at study entry, and all patients were taking systemic prednisolone (40 mg/day). Patients were randomised to receive azathioprine $2 \mathrm{mg} / \mathrm{kg} /$ day or oral 5 -aminosalicylic acid $3.2 \mathrm{~g} /$ day, for a six month follow up period. The outcome of the treatment was defined as (1) success, indicating induction of clinical and endoscopic remission and steroid discontinuation, or (2) failure, indicating the absence of clinical and endoscopic remission and therefore the need for at least one further cycle of systemic steroids to control symptoms, apart from the initial one, or colectomy.

Results: Significantly more patients in the azathioprine than in the 5-aminosalicylic acid group had clinical and endoscopic remission, and discontinued steroid therapy, both in the intention to treat (azathioprine $v$ 5-aminosalicylic acid: 19/36 patients (53\%) v $7 / 36$ (21\%); odds ratio (OR) 4.78 (95\% confidence interval (CI) 1.57-14.5)) and per protocol (azathioprine $\vee 5$-aminosalicylic acid: 19/33 patients (58\%) $\vee 7 / 34$ (21\%); OR 5.26 (95\% Cl 1.59-18.1)) analysis.

Conclusions: Azathioprine is significantly more effective than 5-aminosalicylic acid in inducing clinical and endoscopic remission and avoiding steroid requirement in the treatment of steroid dependent ulcerative colitis.
U lcerative colitis (UC) is a chronic disease characterised by diffuse inflammation of the colonic mucosa associated with a dysregulation of the mucosal immune system. ${ }^{12}$ Most patients present with intermittent chronic disease and recurrent flare ups, with freedom from symptoms in the interim. However, a proportion of patients, ranging from $7 \%$ to $22 \%,{ }^{3-5}$ suffer from chronically active or steroid dependent disease. In this context, therapeutic options include either prolonged systemic corticosteroid therapy or surgical treatment. To reduce the need for steroids and consequent side effects, and more importantly, to avoid colectomy, various alternative pharmacological approaches have been attempted.

Two uncontrolled studies, ${ }^{6} 7$ not yet published in full, have shown that high oral doses of 5-aminosalicylic acid (5-ASA) (from 2.4 to $4.4 \mathrm{~g} /$ daily) can be effective as adjunctive treatment in patients with chronic active steroid dependent UC, reducing or eliminating the need for prednisone in $50 \%$ of patients. However, no controlled trials at present have thus far evaluated the efficacy of 5-ASA for treatment of this group of UC patients.

Given the abnormalities of immune response in the inflamed intestine, immunomodulatory agents such as azathioprine (AZA), 6-mercaptopurine (6-MP), ciclosporin, and methotrexate have been used in selected patients in whom response to steroids was inadequate or when a steroid sparing effect was desired. However, while ciclosporin has been shown to be effective in the treatment of severe refractory $\mathrm{UC},{ }^{8-10}$ the therapeutic role of the remaining immunosuppressive agents is not yet conclusively established. As far as infliximab is concerned, the available data are conflicting, ${ }^{11-16}$ and the results of large, randomised, controlled trials are soon expected.

Considerable experience with the use of AZA and 6-MP in the treatment of IBD has been accumulated since their first use in the 60s. ${ }^{17}{ }^{18}$ However, whereas in Crohn's disease there is evidence that these purine analogues promote both induction and maintenance of remission, ${ }^{19}{ }^{20}$ the efficacy of AZA and 6-MP in UC is still controversial. Several open studies have shown that AZA or 6-MP can be effective in the treatment of patients with $U^{21-26}$ but controlled trials have produced conflicting results. ${ }^{27-33}$

Therefore, we designed a prospective, randomised, controlled study to compare the efficacy and safety of AZA and 5-ASA in inducing remission in steroid dependent UC patients.

\section{METHODS}

A randomised, prospective, investigator-blind, controlled trial, with a parallel group design, comparing the efficacy of AZA (Azatioprina Wellcome; Wellcome Foundation, Italy) with that of 5-ASA (Asacol; Bracco SpA, Milan, Italy) in the treatment of steroid dependent UC, was carried out at the " $\mathrm{L}$ Sacco" University Hospital in Milan, Italy, by medical staff of the gastrointestinal unit, a medical tertiary care centre for the treatment of inflammatory bowel disease.

The study protocol was defined in accordance with the declaration of Helsinki and was approved by the ethics

Abbreviations: AZA, azathioprine; 5-ASA, 5-aminosalicylic acid; UC, ulcerative colitis; 6-MP, 6-mercaptopurine; PGA, physician global assessment; $O R$, odds ratio 
committee of the hospital. Patients gave written informed consent before entering the study.

\section{Patients}

The study population comprised steroid dependent UC patients attending our gastrointestinal unit. To be eligible for the study, patients had to have UC diagnosed by routine clinical, radiographic, endoscopic, and pathological criteria. Steroid dependence was defined as a requirement for a daily dosage of no less than $10 \mathrm{mg}$ of prednisolone and impossibility of weaning the patient off steroid without clinical relapses (two attempts to discontinue the medication within the preceding six months of the start of the study).

At entry, all patients had to have clinically and endoscopically active UC, with a Powell-Tuck index of $\geqslant 8$ and a Baron index of $\geqslant 2$, respectively (see below).

Exclusion criteria included contraindications to the use of 5-ASA or AZA, patients with pre-existing hepatic disease, renal dysfunction, clinically important lung disease, systemic infection, pregnancy, or desire to become pregnant within the next 24 months, breast feeding, history of cancer, hypersensitivity to mesalamine or AZA, erythrocyte macrocytosis, previous use of immunosuppressive drugs and refractoriness to 5-ASA, cytomegalovirus and other infective causes of colitis, and history of steroid resistance, hypertension, glaucoma, diabetes mellitus, and osteoporosis.

On inclusion in the study, age, sex, body weight, smoking habit, disease behaviour, site and duration, UC treatment during the six months prior to inclusion in the study, and mean daily dosage of steroids in the last six months preceding inclusion in the study were recorded for each patient. Previous treatment with oral 5-ASA had to be discontinued at least six months before entry into the trial, and/or topical aminosalicylates, topical corticosteroid, and any other UC specific treatment, except systemic corticosteroids, had to be discontinued at least 15 days before entry into the trial.

\section{Treatment regimens}

Patients who fulfilled the entry/exclusion criteria and who agreed to enter the trial were randomised to receive either AZA or oral 5-ASA by a computer generated list. Randomisation was performed in blocks of 10. AZA was given at a dose of $2 \mathrm{mg} / \mathrm{kg} / \mathrm{day}$ while 5 -ASA was administered at a dose of $3.2 \mathrm{~g}$ per day, divided into three doses $(0.8 \mathrm{~g}$ at breakfast and at lunch and $1.6 \mathrm{~g}$ at dinner).

Simultaneously, all patients received a standard course of prednisolone treatment at the beginning of the trial. A uniform dose was given to control the effects of a primary determinant of disease activity and to provide a common starting point from which to evaluate differences in steroid use between the two groups. The initial dose was $40 \mathrm{mg}$ prednisolone daily for two weeks, then 30 and $20 \mathrm{mg}$ daily, respectively, for the following two and four weeks. After eight weeks, prednisolone was tapered by $5 \mathrm{mg}$ each week until withdrawal if the patient's condition had remained stable or improved. In those patients whose condition worsened, with the occurrence of diarrhoea with blood in the motions, prednisolone dosage was increased to a maximum daily dose of $40 \mathrm{mg}$. After this dose increase, prednisolone was again tapered as described above.

Concomitant use of the following drugs was not allowed during the trial: topical corticosteroids, other immunosuppressive drugs, sulphasalazine, antibiotics (for a cumulative duration of more than 10 days), non-steroidal anti-inflammatory drugs, and other aminosalicylates. Symptomatic treatment with antacids, antidiarrhoeal, or spasmolytic agents was allowed but had to be scrupulously recorded.

\section{Table 1 Modified Powell-Tuck index ${ }^{34}$}

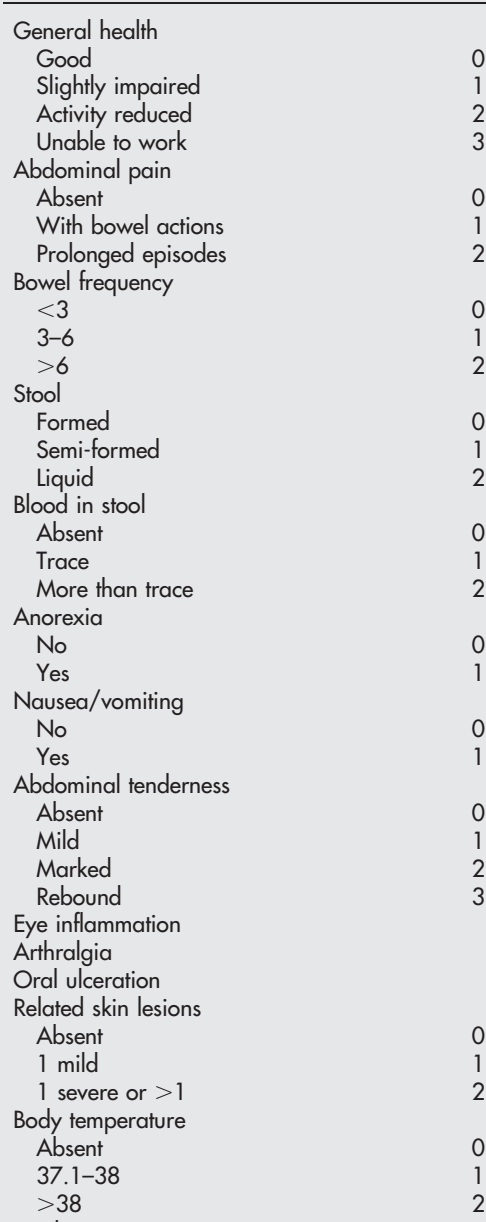

Total

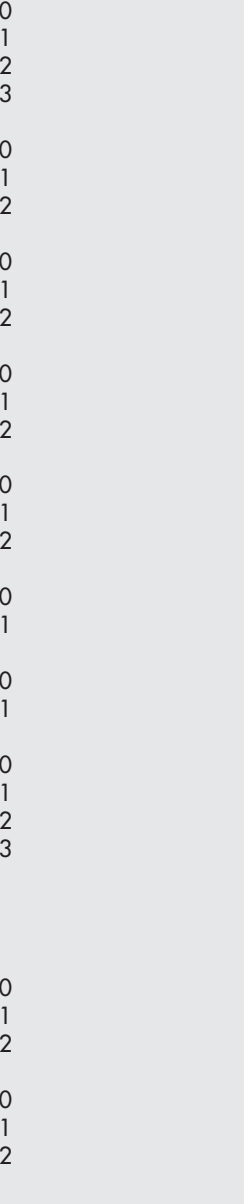

Patients were also requested to precisely record any other concomitant medication in their diary.

Compliance with treatment was evaluated by a simple questionnaire in which adverse events were also recorded.

\section{Study design and follow-up}

Patients and the Head of the Gastrointestinal Unit (GBP), who supervised the randomisation, were aware of the treatment. The principal investigator (SA), who was blinded to treatment assignment, evaluated the clinical and endoscopic efficacy of treatment at each scheduled control and at the end of the study.

The study lasted six months. Patients were clinically evaluated at baseline and every month until the end of the study, while an endoscopic examination was performed at the beginning and after three and six months of therapy. Patients were also evaluated throughout the study period whenever they had a medical problem. Clinical activity was evaluated by a modified Powell-Tuck index (table 1). ${ }^{34}$

This index includes both clinical and endoscopic items. However, in order to monitor endoscopic activity more closely during the study, we scored it separately using the endoscopic index described by Baron and colleagues ${ }^{35}(0=$ normal mucosa; 1 = granular or oedematous with loss of vascular pattern; $2=$ bleeding to light touch; $3=$ spontaneous bleeding ahead of the instrument), considering scores of 0 and 1 as endoscopic remission. The physician global assessment (PGA) $(1=$ very much improved, 
$2=$ much improved, $3=$ minimally improved, $4=$ non change, $5=$ minimally worse, $6=$ much worse) was applied at baseline and after three and six months of therapy.

At each visit, the patient's weight, pulse rate, blood pressure, and body temperature were measured. Patients were asked about symptoms, and scores on the Powell-Tuck index were calculated. The abdomen was palpated and any systemic manifestations of the disease or its treatment were noted. Data on the use of prednisolone were obtained. Blood was taken for full blood count; serum urea, creatinine, electrolytes, serum amylase, and liver function tests were assessed. Urinary protein and glucose levels were also evaluated. Considering the myelo- and hepatotoxicity of AZA, patients receiving this drug were regularly assessed by total blood count and serum transaminases (every three days for the first two weeks, once weekly for the following two weeks, and once a month thereafter for the entire duration of the study) in order to monitor any myelo- and hepatotoxicity of the treatment. The Baron index was computed at each scheduled endoscopic examination.

Patients could be withdrawn from the study for any of the following reasons: non-compliance, severe protracted infection, repeatedly abnormal liver function tests, persistent leucopenia $\left(<3800 / \mathrm{mm}^{3}\right)$, thrombocytopenia $(<100,000 /$ $\mathrm{mm}^{3}$ ), serum aminotransferase increased to three times the upper limit of normal, marked worsening of symptoms, unacceptable side effects, imminent surgery, or patient's request. Patients who discontinued their medication because of adverse events, reactions, or treatment failure were followed in the same way as those who continued to receive therapy.

\section{Evaluation of safety}

Safety was assessed in terms of incidence of adverse events and changes in vital signs and routine laboratory tests. Patients were monitored for adverse events at each scheduled visit.

\section{Outcome measures}

The outcome of the treatment was defined as (1) success, meaning induction of clinical and endoscopic remission and steroid discontinuation or (2) failure, indicating the persistence of symptoms of active disease with a Baron index $\geqslant 2$, and therefore the need for at least one further cycle of systemic steroids to control symptoms, or colectomy, at end of the study. This was considered as the primary outcome parameter for determination of drug efficacy. Clinical remission was defined as absence of symptoms of active disease with a Powell-Tuck index of 0 and endoscopic remission as a Baron index of 0 or 1 .

The following secondary outcome measures were considered: (1) change in mean scores on the Powell-Tuck index; (2) change in mean scores on the Baron index; and (3) change in mean values of the PGA.

Outcome measures were analysed in all randomised patients who had taken at least one dose of the study medication (intention to treat population) as well as in patients adhering to the protocol (per protocol population). The following events were considered protocol violations: failure to return for a follow up visit within two weeks of the scheduled date, lack of compliance (intake of $<80 \%$ of pills), study medication discontinued for a period longer than three weeks, and use of prohibited concomitant medications.

\section{Statistical analysis Sample size}

Our previous survey had shown a probability of remission and discontinuation of systemic steroid of approximately $70 \%$ for patients with steroid dependent UC under AZA. ${ }^{25}$ To show a decrease in remission rate from $70 \%$ to $35 \%$ in those patients taking 5-ASA with a power of 0.8 (two tailed $\alpha=$ $0.05), 72$ patients were required.

\section{Analysis of data}

Homogeneity of the two treatment groups was compared using the Wilcoxon rank sum test for continuous variables and Fisher's exact test for discrete variables. Distribution of individual characteristics was evaluated by simple descriptive statistics.

For efficacy analysis, both intention to treat and per protocol analyses were performed. In the intention to treat analysis, all randomised patients who received at least one dose of the study drug and underwent the baseline evaluation were considered for analysis. All patients who completed the study were included in the per protocol analysis. The proportions of patients with therapeutic success or failure in the two study groups were compared using Fisher's exact test. Failure risk was estimated using odds ratios (OR) and the corresponding 95\% confidence intervals (CI) were calculated from unconditional logistic regression model including age and sex.

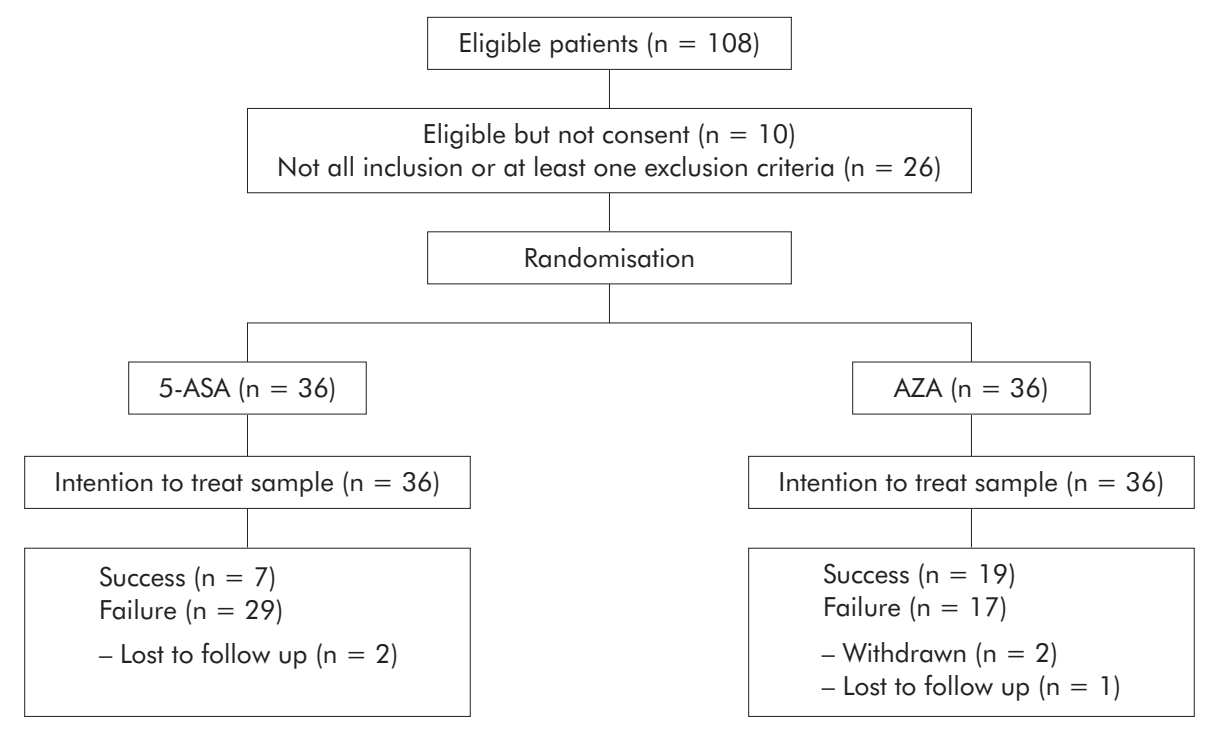

Figure 1 Flow chart illustrating the progress of patients through the study. AZA, azathioprine; 5-ASA, 5-aminosalicylic acid. 


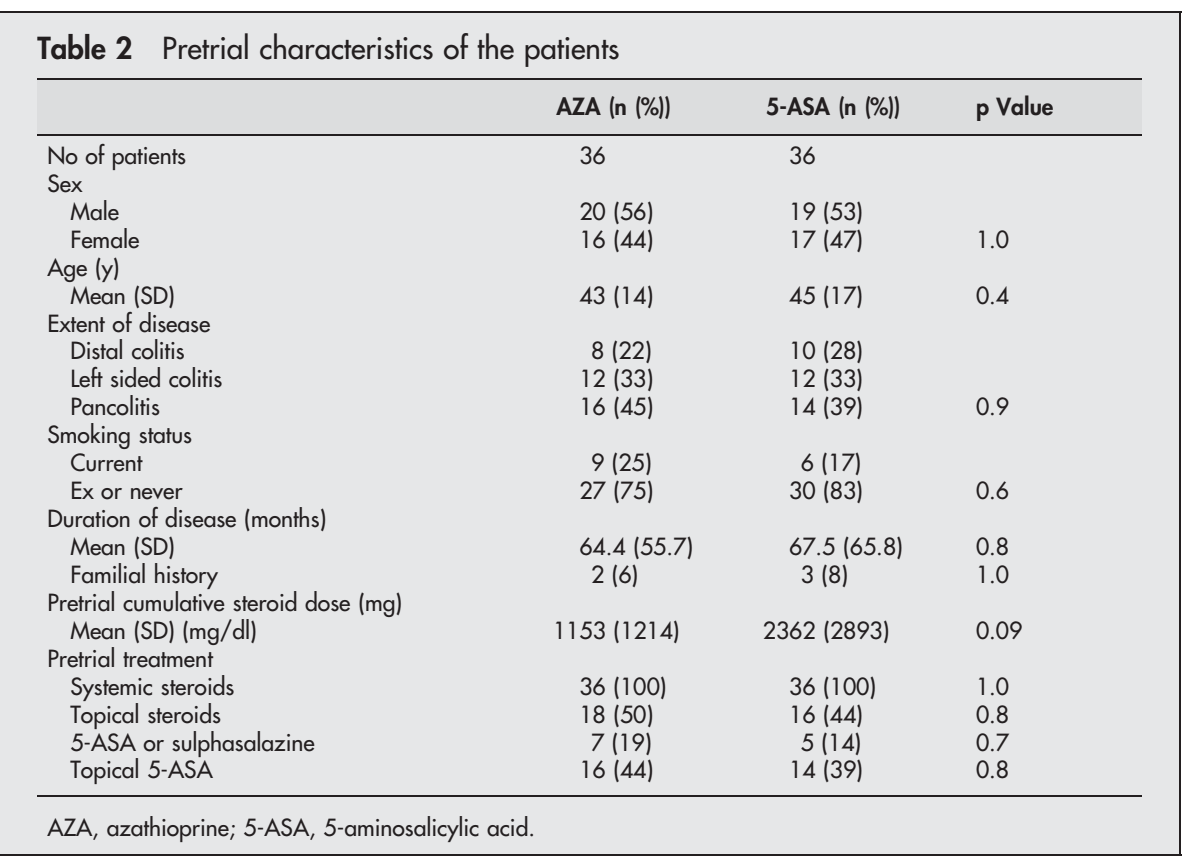

The mean values on the Powell-Tuck and Baron indices and mean values of PGA were evaluated by the Wilcoxon matched pairs signed ranks test for comparison across the single time and between treatments, respectively. All randomised patients who received at least one dose of the study medication and were subjected to the baseline assessment were included. If the score at an assessment time was missing, the last available post-treatment observation was used for the missing assessment point.

Comparisons between treatments were made using two way analyses of variance for repeated measures, with drug treatment as one independent variable and time or occasion as the repeated measures second factor. Two adjustments of F value, proposed by Greenhouse-Geisser and Huynh-Feldt, were used to correct observed significance levels if the test of sphericity of the covariance matrix across the between subjects effects was rejected. Withdrawal from the study was considered as a treatment failure.

The proportion of patients who withdrew from therapy due to adverse reactions, in the two groups, was compared using Fisher's exact test.

All statistical tests were two tailed using a 0.05 level of significance

\section{RESULTS}

\section{Patients}

Patient recruitment began in September 1997 and was completed in April 2000. A total of 108 were screened for the study. Of these, 26 patients were not enrolled because they did not fulfil inclusion criteria and 10 patients refused to take part in the study. Thus 72 patients $(67 \%$ of the eligible population; 27 males, 35 females, mean age 44.16 years (range 18-72)) were randomised, 36 to AZA and 36 to 5-ASA. (fig 1 ).

The pretrial characteristics of the two groups are shown in table 2. No significant difference was observed between the two treatment groups for age, sex, duration of disease, extent and duration of disease, family history, or pretrial treatment, although patients on 5-ASA had a greater pretrial cumulative steroid use $(p=0.09)$. Sixteen out of $36(44 \%)$ and 14 out of $36(39 \%)$ patients treated with AZA and 5-ASA, respectively, discontinued local therapy prior to study entry.

A total of three patients (one on AZA and two on 5-ASA) were lost to follow up while two patients on AZA discontinued treatment due to adverse events. No patients were lost on account of treatment failure, in either the AZA or 5-ASA group.

\section{Efficacy}

Primary outcome parameter

Significantly more patients treated with AZA had clinical and endoscopic remission, and discontinued steroid therapy, in comparison with the 5-ASA group. In particular, in the intention to treat analysis, $19 / 36$ patients (53\%) had therapeutic success in the AZA group compared with $7 / 36$ $(19 \%)$ in the 5-ASA group $(p=0.006$, OR $4.78 \quad(95 \%$ CI

Table 3 Outcome of the study drugs

\begin{tabular}{llllll}
\hline & AZA & 5-ASA & Total & p Value* & OR* (95\% Cl) \\
\hline Intent to treat & $19(53 \%)$ & $7(19 \%)$ & 26 & & \\
$\quad$ Success & $17(47 \%)$ & $29(81 \%)$ & 46 & & $4.78(1.57-14.5)$ \\
$\quad$ Failure & 36 & 36 & 72 & 0.006 & \\
$\quad$ Total & $19(58 \%)$ & $7(21 \%)$ & 26 & & \\
Per protocol & $14(42 \%)$ & $27(79 \%)$ & 41 & 0.003 & $5.26(1.59-18.1)$ \\
$\quad$ Success & 33 & 34 & 67 & & \\
$\quad$ Failure & Total & & & & \\
\hline
\end{tabular}

OR $(95 \% \mathrm{Cl})$, odds ratio (95\% confidence interval).

AZA, azathioprine; 5-ASA, 5-aminosalicylic acid.

*From logistic regression models including terms for age and sex. 


\begin{tabular}{|c|c|c|c|c|c|c|c|c|}
\hline & \multicolumn{3}{|c|}{ 5-ASA } & \multicolumn{3}{|c|}{ Azathioprine } & \multicolumn{2}{|l|}{ p Value } \\
\hline & $n$ & Mean (SD) & Min-Max & $n$ & Mean (SD) & Min-Max & Wilcoxon* & ANOVAt \\
\hline \multicolumn{9}{|c|}{ Powell-Tuck index } \\
\hline Start & 36 & $8.7(2.0)$ & $8.0-13.0$ & 36 & $8.4(1.4)$ & $8.0-11.0$ & 0.4 & \\
\hline 3 months & 36 & $2.8(2.6)$ & $0.0-9.0$ & 36 & $2.6(4.3)$ & $0.0-23.0$ & 0.1 & \\
\hline 6 months & 36 & $2.7(2.9)$ & $0.0-10.0$ & 36 & $1.4-2.6$ & $0.0-11.0$ & 0.01 & 0.3 \\
\hline \multicolumn{9}{|l|}{ Baron index } \\
\hline Start & 36 & $2.6(0.5)$ & $2.0-3.0$ & 36 & $2.6(0.5)$ & $2.0-3.0$ & 0.6 & \\
\hline 3 months & 36 & $2.3(0.8)$ & $1.0-3.0$ & 36 & $1.1(1.2)$ & $0.0-3.0$ & $<0.0001$ & \\
\hline 6 months & 36 & $2.2(1.0)$ & $0.0-3.0$ & 36 & $0.9(1.2)$ & $0.0-3.0$ & $<0.0001$ & $<0.001$ \\
\hline \multicolumn{9}{|l|}{ PGA } \\
\hline Start & 36 & $5.2(0.7)$ & $4.0-6.0$ & 36 & $5.2(0.8)$ & $3.0-6.0$ & 0.8 & \\
\hline 3 months & 36 & $4.6(1.4)$ & $1.0-6.0$ & 36 & $2.6(1.8)$ & $1.0-6.0$ & $<0.0001$ & \\
\hline 6 months & 36 & $4.1(1.8)$ & $1.0-6.0$ & 36 & $2.6(1.9)$ & $1.0-6.0$ & 0.001 & $<0.001$ \\
\hline
\end{tabular}

1.57-14.5)) (table 3). On a per protocol basis, 19/33 patients $(58 \%)$ taking AZA and 7/34 (21\%) on 5-ASA had therapeutic success $(p=0.003$, OR $5.26(95 \%$ CI 1.59-18.1)) (table 3$)$. The absolute risk reduction was 34\% (95\% CI 10.5-57.5) and $37 \%$ (95\% CI 12.3-61.7) for the intention to treat and per protocol analyses, respectively.

Four patients in the AZA and three in the 5-ASA treatment groups underwent colectomy during the study.

\section{Secondary outcome measures}

The mean values of the Powell-Tuck index, Baron index, and PGA at baseline and after three and six months of treatment, according to intention to treat, are shown in table 4. At each time point, differences between treatment are presented. In particular, the Powell-Tuck index decreased from baseline to six months by $84 \%$ and $69 \%(p$ value $=0.009)$, the Baron index by $51 \%$ and $18 \%(\mathrm{p}=0.0001)$, and PGA by $48 \%$ and $19 \%(\mathrm{p}=0.004)$ in the AZA and 5-ASA groups, respectively. Table 4 also show the results from analysis of variance for repeated measures. According to the intention to treat analysis, the Powell-Tuck index showed no statistically significant difference in reduction trend between the two treatment groups. In contrast, the Baron index and PGA showed statistically significant differences in reduction trend between the AZA and 5-ASA groups.

\section{Safety}

Table 5 shows the frequency of adverse events observed during the study and the measures adopted. During the six month follow up period, patients treated with AZA more frequently complained of mild to moderate adverse events than those taking 5-ASA $(26 \% v 6 \%$; $=0.046)$. However, only two patients withdrew from treatment due to adverse

Table 5 Adverse events according to study group

\begin{tabular}{|c|c|c|c|}
\hline & $\begin{array}{l}\text { AZA } \\
(n=36)\end{array}$ & $\begin{array}{l}\text { 5-ASA } \\
(n=36)\end{array}$ & Action \\
\hline Fever and headache & 1 & - & Withdrawal \\
\hline Leuco-thrombocytopenia & 1 & - & Withdrawal \\
\hline Leucopenia & 4 & - & Dose reduction \\
\hline AST/ALT ( $\uparrow)$ & 1 & - & Dose reduction \\
\hline Epigastric pain & 2 & 1 & - \\
\hline Dermatitis & - & 1 & - \\
\hline Total $^{*}$ & $9 / 36(25 \%)$ & $2 / 36(6 \%)$ & \\
\hline
\end{tabular}

AZA, azathioprine; 5-ASA, 5-aminosalicylic acid; AST, aspartate aminotransferase; ALT, alanine aminotransferase. $\uparrow$, once the upper limit of normal.

${ }^{*} p=0.046$. events, all on AZA (one with fever and headache, and one with leuco-thrombocytopoenia). In five patients treated with AZA, a dose reduction was necessary, followed immediately by a return to normal, because of leucopoenia (four patients) and impaired liver function tests (one patient).

\section{DISCUSSION}

In the last decade, significant advances have been made in the therapy of inflammatory bowel disease and new treatments, such as biological therapies, are being successfully introduced. However, while many efforts have been made to improve the management of patients suffering from Crohn's disease, and randomised controlled trials are now available showing that biological agents such as infliximab are effective in inducing and maintaining remission in patients with refractory inflammatory and fistulising Crohn's disease, ${ }^{36-39}$ similar efficacy has not yet been demonstrated in UC.

Thus awaiting the imminent results of large placebo controlled trials of infliximab, the medical treatment of UC still relies on the old conventional therapies. In particular, the great medical need for improved therapy in acute and chronic active corticosteroid resistant and corticosteroid dependent UC remains unsatisfied.

The purine analogues, AZA and its active metabolite 6-MP, are secondline drugs that are increasingly and widely used by gastroenterologists in selected patients with IBD requiring frequent courses of corticosteroids. Several controlled trials and meta analyse ${ }^{2021}$ have established the efficacy of AZA or 6-MP in reducing the need for steroids and maintaining remission, and in treating chronic active disease, in both luminal and fistulising Crohn's disease. In contrast, there is no sound evidence that AZA is effective in the treatment of patients suffering from active UC, especially in steroid dependent and steroid refractory UC. Indeed, the efficacy of AZA treatment in active UC has been evaluated in only five controlled trials, ${ }^{27-30} 32$ most of which were performed approximately 30 years ago, and providing equivocal or negative results. Surprisingly, no other randomised controlled trial has been performed while, after a silence of approximately 20 years, a number of open uncontrolled and retrospective studies have been published ${ }^{21-26}$ in the last decade, all reporting data in favour of AZA or 6-MP as an effective treatment for patients with steroid resistant and steroid dependent UC. Discrepancies among reported studies are probably related to differences in study design (controlled $v$ open label studies), patient population and selection, definition of steroid dependence or steroid resistance, daily dosage and duration of therapy, evaluation of response to 
therapy (clinical $v$ endoscopic outcome), and concomitant treatment with steroids and salicylates.

Bearing in mind these various aspects, we performed a randomised, investigator-blind, controlled trial aimed at comparing AZA and 5-ASA in the treatment of steroid dependent UC. We evaluated the patients included, both clinically and endoscopically, prespecified the primary outcome, and used a strict definition of steroid dependence. Under these conditions, we found that AZA therapy was significantly more effective than 5-ASA in inducing clinical and endoscopic remission, and avoiding steroid requirement in this group of patients, both in the intention to treat and per protocol analyses. In particular, more than $50 \%$ of AZA treated patients had both clinical and endoscopic remission, and discontinued steroid therapy, with a therapeutic gain of approximately $35 \%$ in comparison with 5-ASA. Clinical activity showed a significant decrease in both groups from baseline to six months. In contrast, both endoscopic activity and PGA showed a significant decrease, from baseline to six months, only in AZA treated patients.

With regard to the clinical activity index, comparing the two treatment groups, no significant difference was observed between the two treatment groups after three or six months of therapy. As expected, considering that all patients were treated with systemic steroids, after three months both groups of patients had a similar decrease in clinical activity score. After six months, again, no statistically significant difference was observed between the two treatments. However, while patients treated with AZA were in clinical remission without steroids, patients treated with 5-ASA controlled their symptoms because they were taking another cycle of systemic steroids apart from the initial one. For the endoscopic index, after both three and six month of therapy, AZA was significantly more effective than 5-ASA in inducing endoscopic remission, reducing the endoscopic score to 1 or less in a significantly higher proportion of patients. These findings are very important because it is possible that AZA treated patients achieve and maintain their clinical remission without steroids because of the ability of AZA to induce healing of mucosal lesions. In contrast, patients treated with 5-ASA continue to need systemic steroids to control their symptoms because they fail to achieve endoscopic remission.

An important methodological limitation of the trial was the lack of double blinding, which could have biased the results. Thus considering that patients were aware of the treatment assigned, it is possible that those taking AZA may have believed they were being treated with a more effective drug and were therefore more likely to experience therapeutic success. Moreover, the closer supervision of the AZA patients, regularly assessed in order to monitor the potential myeloand hepatotoxicity of the treatment, could have had some effect on outcome. However, considering that AZA was administered at a weight adjusted dose of $2 \mathrm{mg} / \mathrm{kg} /$ day, performing a double blind double-dummy study would have been very difficult.

In conclusion, the results of this randomised, investigatorblind, controlled trial show that AZA is significantly more effective than 5-ASA in inducing clinical and endoscopic remission, and avoiding steroid requirement in steroid dependent UC. The therapeutic efficacy of AZA may be due to its ability to induce healing of mucosal lesions.

\footnotetext{
Authors' affiliations

S Ardizzone, G Maconi, V Imbesi, E Colombo, G Bianchi Porro, Chair of Gastroenterology, "L Sacco" University Hospital, Milan, Italy A Russo, Epidemiology Unit, Local Health Authority, Milan, Italy Conflict of interest: None declared.
}

\section{REFERENCES}

1 MacDermott RP, Stenson WF. Alterations of the immune system in ulcerative colitis and Crohhn's disease. Adv Immunol 1988;42:285-328.

2 Monteleone I, Vavassori P, Biancone L, et al. Immunoregulation in the gut: success and failures in human diseases. Gut 2002;50(suppl 3):III60-4.

3 Edwards FC, Truelove SC. The course and prognosis of ulcerative colitis. Part I and II: Short term and long-term prognosis. Gut 1963;4:299-315.

4 Farmer RG, Easley KA, Rankin GB. Clinical patterns, natural histpry, and progression of ulcerative colitis. A long-term follow-up of 1116 patients. Dig Dis Sci 1993;38:1137-46.

5 Faubion WA Jr, Loftus EV Jr, Harmsen WS, et al. The natural history of corticosteroid therapy for inflammatory bowel disease: a population-based study. Gastroenterology 2001;121:255-60.

6 Schroeder KW, Tremaine WJ. Corticosteroid dependent chronic ulcerative colitis (CUC): favourable response to oral 5-aminosalycilic acid (Asacol). Gastroenterology 1985;88:A1576.

7 Tremaine WJ, Schroeder KW, Ilstrup DM. The corticosteroid-sparing effect of oral 5-aminosalycilic acid for chronic ulcerative coltis. Gastroenetrology 1987:92:A1673.

8 Lichtiger S, Present DH, Kornbluth A, et al. Cyclosporine in severe ulcerative colitis refractory to steroid therapy. N Engl J Med 1994;330:1841-5.

9 D'Haens G, Lemmens L, Geboes K, et al. Intravenous cyclosporine versus intravenous corticosteroids as single therapy for severe attacks of ulcerative colits. Gastroenterology 2001;120:1323-9.

10 Van Assche G, D'Haens G, Noman M, et al. Randomized, double-blind comparison of $4 \mathrm{mg} / \mathrm{kg}$ versus $2 \mathrm{mg} / \mathrm{kg}$ intravenous cyclosporine in severe ulcerative colitis. Gastroenterology 2003;125:1025-31.

11 Chey WY, Hussain A, Ryan C, et al. Infliximab is an effective therapeutic agent for ulcerative colitis. Am J Gastroenterol 2000;95:A2530-1.

12 Sands BE, Tremaine WJ, Sandborn WJ, et al. Infliximab in the treatment of severe, steroid-refractory ulcerative colitis: a pilot study. Inflammatory Bowel Dis, 2001;7:83-8.

13 Su C, Salzberg BA, Lewis JD, et al. Efficacy of anti-tumor necrosis factor therapy in patients with ulcerative colitis. Am J Gastroenterol 2002;97:2577-84.

14 Actis GC, Bruno $M$, Pinna-Pintor $M$, et al. Infliximab for treatment of steroidrefractory ulcerative colitis. Dig Liver Dis, 2002 Sep, 34:631-4.

15 Kohn A, Prantera C, Pera A, et al. Anti-tumour necrosis factor alpha (infliximab) in the treatment of severe ulcerative colitis: result of an open study on 13 patients. Dig Liver Dis 2002;34:626-30.

16 Probert CS, Hearing SD, Schreiber S, et al. Infliximab in moderately severe glucocorticoid resistant ulcerative colitis: a randomised controlled trial. Gut 2003;52:998-1002.

17 Bean RHD. The treatment of chronic ulcerative colitis with 6-mercaptopurine. Med J Aus 1962;2:592-3.

18 Bowen GE, Irons GV, Rhodes JB. Early experience with azathioprine in ulcerative colitis. JAMA 1966;195:460-4.

19 Sandborn JW, Sutherland L, Perason D, et al. Azathioprine or 6mercaptopurine for induction of remission in Crohn's disease (Cochrane Review). The Cochrane Library. Issue 4. Oxford: Update Software, 2003.

20 Person D, May G, Fick G, et al. Azathiorpine for maintenance remission in Crohn's disease (Cochrane Review). The Cochrane Library. Issue 4. . Oxford: Update Software, 2003.

21 Steinhart AH, Baker JP, Brzezinski A, et al. Azathioprine therapy in chronic ulcerative colitis. J Clin Gastroenterol 1990;12:271-5.

22 Lobo AJ, Foster PN, Burken DA, et al. The role of azathioprine in the management of ulcerative colitis. Dis Colon Rectum 1990;33:374-7.

23 Adler DJ, Korelitz BI. The therapeutic efficacy of 6-mercaptopurine in refractory ulcerative colitis. Am J Gastroenterol 1990;85:717-22.

24 George J, Present DH, Pou R, et al. The long-term outcome of ulcerative colitis treated with 6-mercaptopurine. Am J Gastroenterol 1996:91:1711-14.

25 Ardizzone S, Molteni P, Imbesi V, et al. Azathioprine in steroid-resistant and steroid-dependent ulcerative colitis. J Clin Gastroenterol 1997;25:127-30.

26 Fraser AG, Orchard TR, Jewell DP. The efficacy of azathioprine for the treatment of inflammatory bowel disease: a 30 year review. Gut 2002;50:485-9.

27 Jewell DP, Truelove SC. Azathioprine in ulcerative colitis: final report on controlled therapeutic trial. BMJ 1974;4:627-30.

28 Caprilli R, Carratu R, Babbini M. A double-blind comparison of the effectiveness of azathioprine and sulphasalazine in idiopatic proctocolitis. Dig Dis 1975;20:115-20.

29 Rosenberg JL, Wall AJ, Levine B, et al. A controlled trial of azathiorpine in the management of chronic ulcerative colitis. Gastroenterology 1975;69:96-9.

30 Kirk AP, Lennard-Jones JE. Controlled trial of azathioprine in chronic ulcerative colitis. BMJ 1982;284:1291-2.

31 Hawthorne AB, Logan RFA, Hawkey CJ, et al. Randomised controlled trial of azathioprine withdrawal in ulcerative colitis. BMJ 1992;305:20-2.

32 Sood A, Midha V, Sood N, et al. Role of azathioprine in severe ulcerative colitis: one-year, placebo-controlled, randomised trial. Indian J Gastroenterol 2000;19:14-16.

33 Mantzaris GJ, Sfakianakis $M$, Archavlis E, et al. A prospective randomized observer-blind 2-year trial of azathioprine monotherapy versus azathioprine and olsalazine for the maintenance of remission of steroid-dependent ulcerativie colitis. Am J Gastroenterol 2004;99:1122-8.

34 Powell-Tuck J, Bown RL, Lennard-Jones JE. A comparison of oral prednisolone given as single or multiple daily doses for active proctocolitis. Scand J Gastroenterol 1978;13:833-7.

35 Baron JH, Connell AM, Lennard-Jones JE. Variation between observer in describing mucosal appearances in proctocolitis. BMJ 1964;i:89-92. 
36 Targan SR, Hanauer SB, van Deventer SJ, et al. A short-term study of chimeric monoclonal antibody CA2 to tumor necrosis factor alpha for Crohn's disease. Crohn's Disease cA2 Study Group. N Engl J Med 1997;337:1029-35.

37 Present DH, Rutgeerts P, Targan S, et al. Infliximab for the treatment of fistulas in patients with Crohn's disease. N Engl J Med 1999;340:1398-405.
38 Hanaver SB, Feagan BG, Lichtenstein GR, et al. Maintenance infliximab for Crohn's disease: the ACCENT I randomised trial. Lancet 2002;350:1541-9.

39 Sands BE, Anderson FH, Bernstein CN, et al. Infliximab maintenance therapy for fistuliting Crohn's disease. N Engl J Med 2004;350:876-85.

\section{EDITOR'S QUIZ: GI SNAPSHOT}

\section{A rare cause of diuretic refractory ascites}

\section{Clinical presentation}

A 63 year old woman was admitted for diuretic refractory ascites. Her mother was affected by chronic renal failure due to polycystic kidney disease and she suffered from arterial hypertension. Ten months before she had presented with abdominal distension; laboratory tests showed serum creatinine $2 \mathrm{mg} / \mathrm{dl}$, normal liver function, negative hepatitis $\mathrm{B}$ surface antigen, and hepatitis $\mathrm{C}$ virus antibody. Abdominal ultrasound revealed ascites, hepatomegaly, multiple hepatic and renal cysts, and patent portal and hepatic veins. Laparoscopy excluded cirrhosis or malignancy and upper gastrointestinal endoscopy was normal. Diuretic therapy and a low sodium/protein diet were prescribed but the abdominal distension worsened and required repeated paracenteses. At admission, the patient was confined to bed, her appetite and nutritional condition were poor; physical examination disclosed tender hepatomegaly, tense ascites, and mild lower limb oedema. Liver tests were normal, serum albumin $3.0 \mathrm{~g} / \mathrm{dl}$, creatinine $2.4 \mathrm{mg} / \mathrm{dl}$, urea $102 \mathrm{mg} / \mathrm{dl}$, serum electrolytes normal, creatinine clearance $18 \mathrm{ml} / \mathrm{min}$, haemoglobin $10.8 \mathrm{~g} / \mathrm{dl}$, and platelets $190000 / \mathrm{mm}^{3}$. The serum-ascites albumin
Robin Spiller, Editor

gradient was $2.0 \mathrm{~g} / \mathrm{dl}$. Cytology and microbiological investigations were negative. Ascites was unresponsive to furosemide at a dose of $150 \mathrm{mg} /$ day and recurred quickly after paracentesis.

\section{Question}

Abdominal magnetic resonance imaging and cavography images (fig 1) are depicted. What is the diagnosis?

See page 73 for answer

This case is submitted by:

G G Di Costanzo

Division of Hepatology, Cardarelli Hospital, Napoli, Italy A Ragozzino, R Niola, F Maglione Department of Vascular and Interventional Radiology, Cardarelli Hospital, Napoli, Italy

A Ascione

Division of Hepatology, Cardarelli Hospital, Napoli, Italy

Correspondence to: Dr Giovan Giuseppe Di Costanzo, Division of Hepatology, Cardarelli Hospital, Via GB Ruoppolo 59, 80128 Napoli, Italy; bfbdic@tin.it

doi: $10.1136 /$ gut.2005.067868

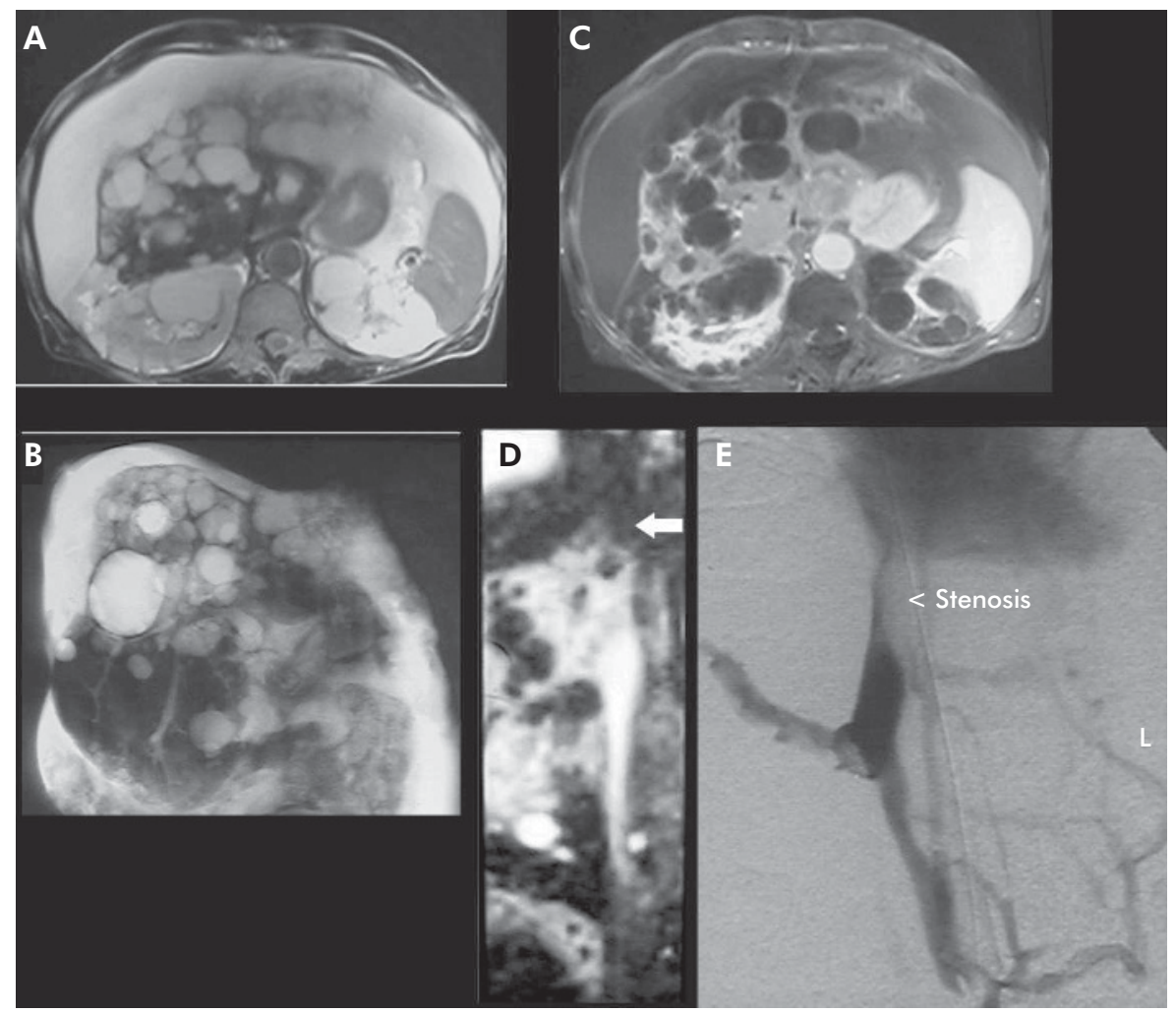

Figure 1 Magnetic resonance imaging (MRI) axial and coronal T2 weighted images (A, B). MRI gadolinium enhanced T1 weighted image (C). Sagittal plane reconstruction of image (D). (E) Cavogram. 